\title{
Salim-Eisa method for modification of evaporation test (British pharmacopeia) by Sudanese essential oils
}

\begin{abstract}
The experiment was conducted in Processing Department, Food Industries Institute, Industrial Research and Consultancy Centre, Khartoum, Sudan to modify Evaporation Test Method of Essential Oil time from 24hours as in British Pharmacopeia to 5-50minutes. Oils extracted from five chosen Sudanese essential oils bearing plants namely Boswellia sp. (Olibanium), Cympobogon citraus (Lemon grass), Mentha spicata (Spearmint), Eucalyptus camaldulensis (Camphorl), Cympobogon proximis (Camel's hay) and one imported Clove buds (Syzygium aromaticum) by hydro distillation according to British Pharmacopeia. The 6 oils were spotted (2mg from each) in filtered papers to form greasy spot and enforced to evaporate under airforced oven at temperatures of $60^{\circ} \mathrm{C}, 70^{\circ} \mathrm{C}, 80^{\circ} \mathrm{C}, 90^{\circ} \mathrm{C}$ and $100^{\circ} \mathrm{C}$ consequently. The results obtained showed that the volatilization time was decreased with an increase in temperature from $60^{\circ} \mathrm{C}$ to $100^{\circ} \mathrm{C}$. Evaporation time at $60^{\circ} \mathrm{C}$ was ranging between 30 50 minutes according to the nature of the essential oil. The minimum evaporation time of essential oil was recorded at $100^{\circ} \mathrm{C}$ range in a period of just $5-15$ minutes depending on the oil obtained from different plants. In conclusion under all temperature levels of evaporation the greasy spots of essential oil were disappeared by evaporation and the greasy spot of fixed oil remained.
\end{abstract}

Keywords: evaporation test, essential oil, olibanium, lemon grass, spearmint, eucalyptus, clove, camel's hay
Volume 3 Issue 2 - 2017

\author{
El Rasheed Ahmed Salim, Maawia \\ Mohammed Eisa \\ Industrial Research and Consultancy Centre, Sudan
}

Correspondence: El Rasheed Ahmed Salim, Industrial Research and Consultancy Centre, Ministry of Industry, Sudan, Tel 002499|2204672,Email rasheedahmedsalim@hotmail.com

Received: September 20, 2016 | Published: June 01, 2017

\section{Introduction}

Essential oils (volatile or ethereal oils) are the odorous principles found in various plant parts and; as their names imply; are volatile in steam. ${ }^{1}$ These volatile odoriferous constituents differ in both chemical and physical properties from fixed oils. They occur in many plants as organic compounds of various complexity including hydrocarbons, monoterpenes and sesquiterpenes, alcohols, ketones, aldehydes, esters, ethers and etc. ${ }^{2}$ Essential oil characterized by readily evaporation when exposed to air at normal room temperature. ${ }^{2}$ Volatile oil evaporates completely from filter paper without leaving a translucent or greasy mark onto a filter paper. ${ }^{3}$ During the drying process the condition or quality of spearmint changes and result in loss of essential oil. ${ }^{4}$ The essential oil constituents can be adversely affected during drying of herbs. ${ }^{4,5}$ Drying in a convection oven resulted in losses of volatile compounds that fluctuated with the drying temperature and drying time employed. ${ }^{6}$

Adulteration is a very tedious processes that headache the consumer of essential oils. There are different adulterations and different type of tests to investigate adulteration. Blending-in cheaper oils to meet a customer's target purchasing price or to make additional profit for the producer is common place in the oil trade. Some practices mentioned by Arctander for example, the practice of extending of Amyris oil (Amyris balsamifera) with Cedarwood oil Virginia (Juniperus virginiana) \& Copaiba Balsam (Copaifera spp.) are unlikely to fool too many potential customers in these present \& more educated times, but other more common adulteration practices still remain, ${ }^{7}$ for example; addition of isolates like eugenol \& eugenyl acetate to clove bud oil. Addition of single raw materials in adulteration can be conveniently divided into two groups: "Invisibles" i.e. those materials undetectable by a gas chromatograph (GC) analysis operating under routine set conditions employed to analyse essential oils or "Visibles those materials which are normally detectable by GC. "Invisibles": an example of this type is the deliberate addition of vegetable or mineral oil to essential oils. ${ }^{8}$ Theoretically the "total area" of the detectable components of the oil's gas chromatogram should be reduced by this latter type of adulteration, creating a suspicious situation for the analyst prompting the need for further investigation. These adulterant materials may be revealed by simple aqueous alcohol solubility tests and their presence further verified by using a different GC column and operating conditions (to detect mineral oil), or by derivatization (for example the use of a methylating agent to react with the vegetable oil, thereby creating volatile methyl esters of the fatty acid components of oil's glyceryl esters, which can be revealed by subsequent GC analysis).

Identification of essential oil proprieties is an important factor that determines the oil quality and to avoid adulteration. On the other hand, minimization of evaporating time that required to performing purity test by evaporation test is a vital issue. Since the old test used to identify the purity of essential oil from fixed oil takes about 24 hour, ${ }^{9}$ or as LisBalchin ${ }^{10}$ report that identification of adulteration using fixed oils is simple by a drop of the sample on blotting paper or a piece of paper or cloth will show a halo of grease on evaporation of the essential oil, usually after a few hours. Pure essential oils evaporate completely. ${ }^{10}$ The evaporation temperature and its boundary conditions, from the point view of the heat and mass transfer. Mass transfer normally depends on the nature and localization of liquid and vapor within the plant as a porous medium. It may be conducted by diffusion 
reinforced by capillary force for liquid/solid interaction and by a similar DARCY process within the holes for gas/solid interaction. ${ }^{11}$ In steam extraction, Hasatani et al. ${ }^{12}$ assumed that the mass transfer is a gas phase diffusion, which can be shown as a Fick-type law related to the partial pressure gradient of each volatile compound through an effective diffusivity Deff. Thus, the kinetics depends on several factors such as the compound nature and thermodynamic properties, the temperature, structure and morphological state of the plant (degree of porosity, specific surface area, permeability of secretion element walls etc.). It was found to be highly effective enabling a considerable reduction in evaporation time by convection and conduction due to heat and air velocity, providing an essential oil with a chemical composition enriched in different oil compounds but with yields similar behavior to those of steam distillations during evaporation. There are three main energy transfer govern the evaporation process.

\section{Radiation}

Radiant energy travels uninterrupted until it strikes a surface where it is absorbed, reflected or transmitted. Radiation is an exchange of energy between two surfaces at two different temperatures without contact and transfer medium via emission, reflection and absorption of thermal radiation. ${ }^{11}$ The radiation gives up energy to heat materials when it is absorbed. The rate of heat transfer will depend on

i. Surface temperatures of the heating and receiving materials

ii. The surface properties of the two materials and

iii. The shape of the emitting and receiving bodies.

The amount of heat emitted from a perfect radiator (black body) is calculated using the Stefan-Boltzmann equation:

$$
Q r=\sigma A T^{4}
$$

Where $\sigma$ is the Stefan-Boltzmann constant and $\mathrm{A}$ is the area. Radiant heaters are not perfect. To take this into account, the concept of gray bodies is used and the equation is modified accordingly:

$$
Q r=\varepsilon \sigma A T^{413}
$$

Where $\varepsilon$ is emissivity of the gray body (scale from 0 to 1 ). For most food products, the emissivity is equal to the absorptivity. The net heat transfer by radiation to oil is determined by

$$
Q r=\varepsilon \sigma A\left(T^{4} 1-T^{4} 2\right)^{14}
$$

Where $\mathrm{T} 1$ is the temperature of the emitting body surface and $\mathrm{T} 2$ is the temperature of the oil sample.

\section{Convection}

Convection heat transfer is a result of the movement of molecules or particles either by density gradients set up by temperature differences or by forced movement of the particles by external means such as a fan. Convection is governed by Newton's law which states that the rate of convective heat transfer is proportional to the surface area of heat transfer and the temperature difference between the surface and the fluid:

$$
Q c v=h A\left(T_{s}-T\right)^{9}
$$

Where Qcv is the heat transfer by convection, $\mathrm{h}$ is the heat transfer coefficient, $\mathrm{T}_{\mathrm{s}}$ is the product surface temperature and $\mathrm{T}$ is the temperature of the bulk fluid. ${ }^{11}$
Thermal boundary layer: The thermal boundary layer is considered as an insulating layer which resists heat flow between the air mass and the product surface. This boundary layer has different properties and a different velocity from the main fluid mass. ${ }^{15}$ Since the convective heat transfer coefficient is normally defined based on air mass temperature outside the boundary layer, in order to calculate the heat transfer coefficient, the air temperature outside the boundary layer must be known. An easy way to determine is to position various thermocouples as different height from the surface of the product. ${ }^{11}$

\section{Conduction}

Conduction heat transfer occurs in solid bodies and is transmitted directly through molecules. The rate of heat transferred by conduction is determined by the temperature difference between the oil and the heating/cooling medium and the total resistance to heat transfer. Fourier's law of heat conduction states that the heat transfer rate through a uniform material is directly proportional to the area of heat transfer and the temperature gradient with reference to the thickness in the direction of heat transfer. Mathematically, for 1D heat transfer:

$$
d Q c d t=-k A d T d x^{16}
$$

Where $\mathrm{d} \mathrm{Qc} / \mathrm{dt}$ is the rate of heat transfer, A is area of heat transfer, $\mathrm{dT} / \mathrm{dx}$ is the temperature gradient across unit thickness and $\mathrm{k}$ is the proportionality constant (thermal conductivity). A negative sign is included on the right-hand side because $\mathrm{dT} / \mathrm{dx}$ is negative (temperature decreases as thickness increases). ${ }^{11}$ Taking into consideration the above principals and because of that 24 hours is too long time to have a result in detecting adulteration of essential oil by vegetable oil (triglyceride fatty acids molecules), it is a must under globalization to minimize the testing time. Therefore, shortening the time to reach the final results in few minutes is a vital aspect. The aim of this study is to modify the evaporation test from 24 hour to 5-50 min, depending on the essential oil such as; Olibanium sp, Cympobogon citratus, Mentha spicata, Eucalyptus camaldulensis, Clove and Cympobogon proximis; volatility, using air-forced oven adjusted to different temperature levels namely $60^{\circ} \mathrm{C}, 70^{\circ} \mathrm{C}, 80^{\circ} \mathrm{C}, 90^{\circ} \mathrm{C}$ and $100^{\circ} \mathrm{C}$.

\section{Materials and methods}

\section{Source of samples}

Three samples from oil bearing plants; Olibanium, Clove buds and Camel's hay were obtained from Oumdurman, Sudan local market. Clove buds sample was imported to Sudan but their essential oil extracted in Sudan. Lemon grass and Eucalyptus samples were obtained from demonstration farm, Khartoum North, IRCC, Sudan. Spearmint sample was obtained from Hafaya area, Khartoum North, Sudan.

\section{Oil extraction}

Oil was extracted by water distillation according to the technique of British Pharmacopoeia. ${ }^{9}$ Fifty grams of dried samples were taken and placed in a $2000 \mathrm{ml}$ round bottomed flask. One liter of water was added; so that the sample was completely immersed. The flask was adjusted to Clevenger apparatus equipped with a condenser. Flask contents were brought to boiling for two hours using electric heating mantle. The mixture of water and volatile oil vapor were affected to evaporate, condense and collected as two layers in the distillation apparatus. After distillation was completed, the whole system was left undisturbed for about half an hour so that a good separation was 
obtained (oil/water layers). The volume of the oil was determined and expressed in $\mathrm{ml}$ per 100gram dry weight.

\section{Evaporation test procedure}

Two drops of each essential oils (2mg) were dropped on a filter paper MN 640w 9cm Ǿ manufactured by Macherey-NAGL+Co-516 DURE N-Germany. The filter papers of different oil drops were placed separately in air-forced oven (BTL-BS 2648, WATS 230/250, AMPS 13, Cycles 50/60, No 142021/32, manufactured by Baird and Tatlock (London) LTD, Chadwell Heath Essex England); and subjected to different levels of temperature; $60^{\circ} \mathrm{C}, 70^{\circ} \mathrm{C}, 80^{\circ} \mathrm{C}, 90^{\circ} \mathrm{C}$ and $100^{\circ} \mathrm{C}$. After every 2 minutes the transparency spot diameter of each filter paper was recognized till complete evaporation of essential oil occurred. Times of evaporation for each essential oil; from the different plant species at the different levels of temperature were recorded. A filter paper spotted with vegetable oil was added to the oven with each treatment as a blank treatment and the greasy appearance of volatile and fixed oil dispersion and enlargement were noticed.

\section{Results and discussion}

\section{Evaporation time}

The results showed that all the essential oils at $60^{\circ} \mathrm{C}, 70^{\circ} \mathrm{C}, 80^{\circ} \mathrm{C}$, $90^{\circ} \mathrm{C}$ and $100^{\circ} \mathrm{C}$ levels of temperatures evaporated from filter paper without remaining of any greasy "spot" this indicate that there is no adulteration of essential oil with fixed oil and it is a pure essential oil. Also the results of the minimum and maximum time for evaporation of essential oil; for the 6 essential oils; from filter paper under different temperature levels of air-forced oven has been illustrated in Figure 1. The results showed that the evaporation rate was increased with an increase of temperature from $60^{\circ} \mathrm{C}$ to $100^{\circ} \mathrm{C}$. The minimum time for evaporation at $60^{\circ} \mathrm{C}$ was noticed in 30 minute and maximum time of the evaporation was 50 minute. The minimum time of evaporation at $70^{\circ} \mathrm{C}$ was noticed in 15 minute and the maximum time was 27 minute. The minimum time of evaporation at $80^{\circ} \mathrm{C}$ was noticed in 8 minute and the maximum time was 19 minute. The minimum time of evaporation at $90^{\circ} \mathrm{C}$ was noticed in 6 minute and the maximum time was 17 minute. Minimum time for evaporation at $100^{\circ} \mathrm{C}$ was 5 minute and the maximum time was 15 minute. These findings postulated that the time for evaporation of essential oil from filter paper is inversely affect by air-force oven temperature; and this agree with Salim who found that oil content of spearmint essential oil decreased with an increase in oven temperature. ${ }^{17}$ Nevertheless, essential oil composition and quality are greatly influenced by oven drying temperature and about $75.7 \%$ of essential oils are lost during oven drying at $60^{\circ} \mathrm{C}$ than at $30^{\circ} \mathrm{C} .18$

This agrees with the principals of Marcotte..$^{11}$ Located at the end of the processing line, heating is essential to accelerate evaporation of essential oil. Evaporation is a very complex unit operation leading to products undergoing numerous physical and chemical changes, such as air and oil phase transition, oil evaporation and denaturation of oil. These changes are appreciable involving as well; these will happen as a consequence of simultaneous heat and mass transfers within the product and with the environment inside the oven. Heat transport to the product will occur through radiation from oven walls and product surfaces, natural and forced convection from the movement of hot air, conduction from the carrier medium and within the filter paper. Oil transfer will occur through the evaporation of oil from the filter paper to the air and may also occur as a result of direct gas burning within the oven. Depending upon filter paper essential oil characteristics and oven type, the relative contribution of the various mechanisms of heat transfer can be adjusted to influence the oil transport in order to achieve a desired quality in the final results. Any attempt to modify or alter the process requires a thorough understanding of heat and mass transfer phenomena upon evaporation and their relationship with physical, chemical and biochemical changes involved. ${ }^{11}$

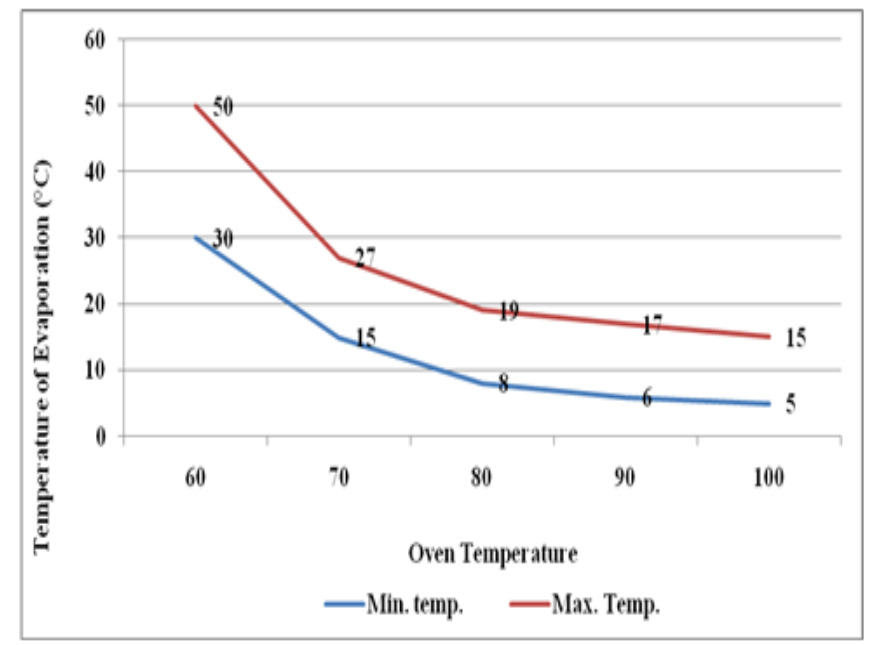

Figure I Minimum and maximum evaporation temperature of some essential oils from filter paper.

\section{Evaporation of different essential oils}

Figure 2 showed the differences in evaporation of different essential oils from different plant species at $100^{\circ} \mathrm{C}$. The result showed that the Olibanium oil minimum evaporation temperature was at $5^{\circ} \mathrm{C}$, Lemon grass was at $5.4^{\circ} \mathrm{C}$, Mint was at $6.2^{\circ} \mathrm{C}$, Eucalyptus was at $8.2^{\circ} \mathrm{C}$, Clove was at $11.2^{\circ} \mathrm{C}$ and Camel's hay was the base one evaporate at $15^{\circ} \mathrm{C}$. It was clearly that the different plants oils evaporate at different times. This because the differences in oil composition from one plant to another. Moreover, each oil component has their own physiochemical properties then performs different levels of volatility. This is due to that the different oils contain different oil components of different chemical functional groups have different ecological behavior. Due to the drying conditions, the rate of loss of different oil components may be different. This agree with that lighter oil components may be evaporated at higher rates than the heavier components, ${ }^{19}$ that can resulted in total loss of essential oil. ${ }^{4}$ Artemisinin oil content in Artemisia annua L. (Asteraceae) was retained to a greater extent, when plants were dried under ambient temperature compared to sundrying or forced-air at 300 to $0^{\circ} \mathrm{C}$ it behave differently. ${ }^{20}$ Diaz-Maroto et al. ${ }^{21}$ evaluated the effect of drying method on volatile compounds content, structural integrity and sensory characteristics of parsley and found that conventional oven-drying resulted in losses of volatile compounds that fluctuated with the drying temperature and drying time employed.

Evaporation of different essential oils at $60^{\circ} \mathrm{C}$ illustrated in Figure 3 . It explained the maximum volatilization temperature of different essential oils. The minimum time of evaporation at $60^{\circ} \mathrm{C}$ was noticed in 30 minute for Olibanium, Lemon grass $34^{\circ} \mathrm{C}$, Mint $37^{\circ} \mathrm{C}$, Eucalyptus $39^{\circ} \mathrm{C}$, Clove $46^{\circ} \mathrm{C}$ and maximum time was in 50 minute for Camel's hay. The different volatility of essential oil in different species was due to the differences in the oil components. The volatility of essential oil 
compounds agree with Bartly et al. ${ }^{5}$ who mentioned that the essential oil constituents can be adversely affected during drying of herbs ${ }^{5}$ and also with Diaz-Marotom et al. ${ }^{21}$ found that the drying method had an effect on the volatile compounds. ${ }^{5}$ Deans and Svoboda mentioned that loss of essential oil is unavoidable during drying processe. ${ }^{22,23}$ This postulation agrees with Paul Boizot ${ }^{24}$ who stated that essential oils evaporate at different rates. Aromatherapy has borrowed the perfumer's terminology and describes oils that evaporate quickly as "top notes", those that evaporate slowly as "base notes" and those inbetween as "middle notes". This is not the same as whether oil has a strong or weak odour, though the two things can be confused, as oil that evaporates quickly will have a greater immediate impact on your sense of smell. Lemongrass is TOP NOTES, peppermint is TOP TO MIDDLE and clove is BASE.

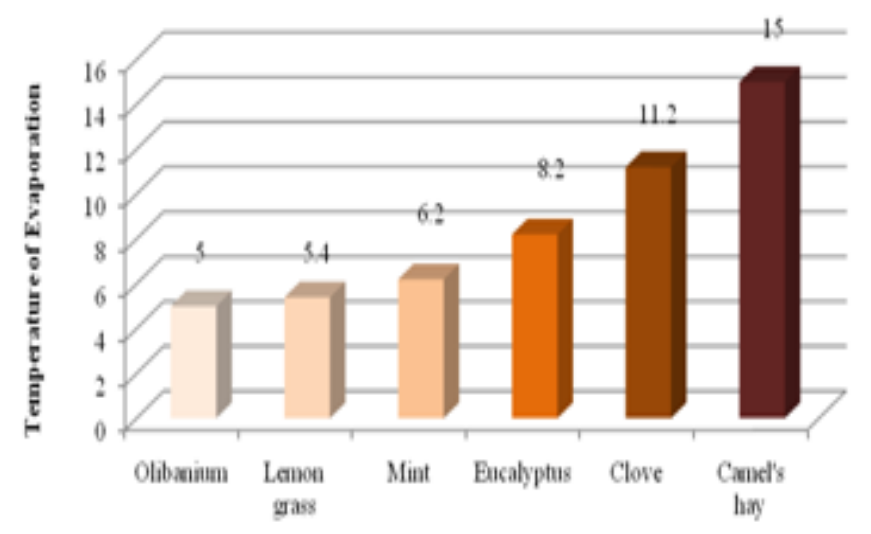

Pant Spedes

OLbanium Lemongass "Mint =Êcalyptus aClove -Canel'shy

Figure 2 Evaporation of essential oil of some plant species from filter paper at $100^{\circ} \mathrm{C}$.

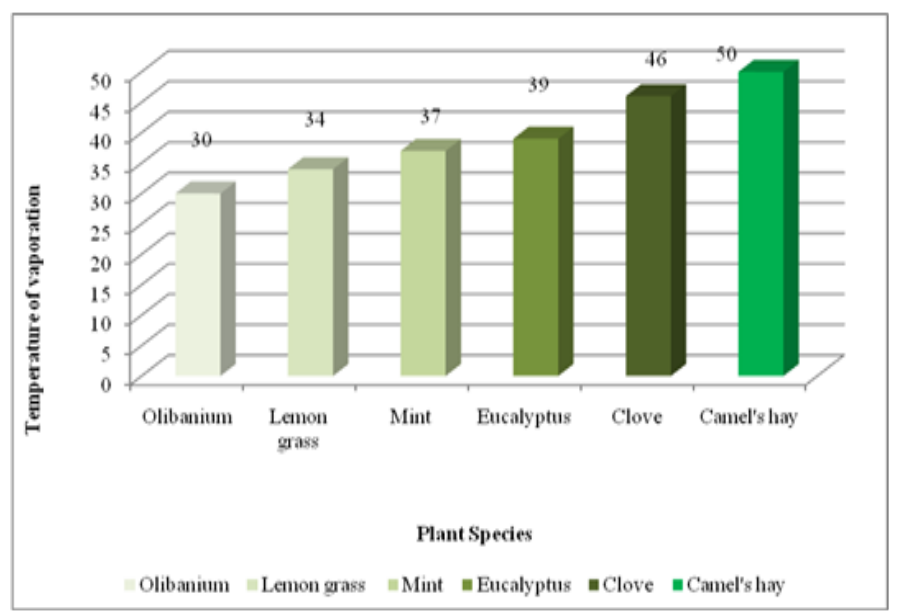

Figure 3 Evaporation of essential oil of some plant species from filter paper at $60^{\circ} \mathrm{C}$.

\section{Fixed oil fixation}

The results showed appearance of fixed vegetable oil (Groundnut oil) greasy spot under all temperature from $60^{\circ} \mathrm{C}, 70^{\circ} \mathrm{C}, 80^{\circ} \mathrm{C}, 90^{\circ} \mathrm{C}$ and $100^{\circ} \mathrm{C}$. The nature of vegetable oil is triglyceride ester fatty acids and differs in their nature from essential oils. But its greasy appearance tend to be slight, more dispersion and enlargement of fixed oil spot under high temperature; this results agree with British Standard Institute specifications which illustrated that in using refractive index correction factors under high temperature more than $20^{\circ} \mathrm{C}$ will be added to the essential oil refractive index value, since temperature increase the spread value of fixed oils ${ }^{3}$ due to an increase in physical properties of the oil. These agree with what mentioned by British Pharmacopeia fixed oil greasy appearance in filter paper does not disappear by volatilization. ${ }^{9}$

\section{Conclusion}

We can conclude that using of adopted Salim et al. ${ }^{17}$ Method by spotting 2 drops of essential oil on filter paper and place in airforced oven for a temperature of $100^{\circ} \mathrm{C}$ are quite enough to complete evaporation test of adulteration of essential oil within 5-15 minutes and at a temperature of $60^{\circ} \mathrm{C}$ are quite enough to complete evaporation test of adulteration in essential oil within 30-50 minutes depending on the essential oil chemical composition; instead of evaporation test under normal temperature for 24 hours.

\section{Ethical approval}

This work is done by authorized people for IRCC and Sudan government. This work is suitable just to identify the adulteration of fixed oil for foods or pharmaceutical purposes. But the other identification test i.e. for heavy metal, other chemical adulteration and other adulteration can be conducted to supplement this work for other adulterations. But this work can substitute methylation process for column separation process of fatty acids when injected in $\mathrm{GC}$ to investigate adulteration of essential oil by fixed ones. It is quite enough for fix oil inspection and you can precede GC process without methylation after this test. It is suitable for Institutional, State, National or International Committee. And it is a legislation work.

\section{Acknowledgements}

None.

\section{Conflict of interest}

The author declares no conflict of interest.

\section{References}

1. Geunther E. The essential oils. 5th ed. New Jersey, USA: Van Nostard Company; 1949.

2. Abu Zeid AN. Aromatic Plants and their Agricultural and Pharmaceutical Products. Al Dar Al Arabia for Printing and distribution. 1st ed. Cairo, Egypt; 1992. 473 p.

3. Methods of Test for Essential oils. London, UK: British Standard Institution; $1976.28 \mathrm{p}$.

4. Ozcan M, Arslan D, Unver A. Effect of drying methods on the mineral content of basil (Ocimum basilicum L.). Journal of Food Engineering. 2005;69(3):375-379

5. Bartley JP, Jacobs AL. Effects of drying on flavour compounds in Australian grown ginger (Zingiber officinale). J Sci Food Agric. 2000;80(2):209-215.

6. Díaz-Maroto M, Pérez-Coello M, Cabezudo M. Effect of different drying methods on the volatile components of parsley (Petroselinum crispum L.). Eur Food Res Technol. 2002;215(3):227-230. 
7. Steffen A. Flavouring essences and essential oils perfumes. New Jersey, USA: Elizabeth; 1960.

8. Nour-el-Din H, Osman AE, Higazy S, et al. The adulteration of essential oils. Egyptian Journal of Food Science. 1977;5(1/2):67-77.

9. British Pharmacopeia. General Medicinal Council. London, UK: The Pharmaceutical Press; 1968.

10. Lis-Balchin M. Aromatherapy Science. London, UK: Pharmaceutical Press; 2005.

11. Marcotte M. Heat and mass transfer during baking. WIT Transactions on State-of-the-art in Science and Engineering. 2017;13:27.

12. Hasatani M, Arai N, Katsuyama H. Heat and mass transfer in bread during baking in an electric oven. In: Mujumdar AS, et al. editors. Netherlands: Elsevier Science Publishers; 1991. p. 385-393.

13. Carvalho MG, Martins N. Mathematical modeling of heat and mass transfer in a forced convection baking oven. AIChE Symposium Series-Heat Transfer, San Diego, USA; 1992. p. 205-211.

14. Falhoul D, Trystram G, McFarlane I, et al. Measurements and predictive modelling of heat fluxes in continuous baking ovens. $J$ food eng. 1995;26:469-479.

15. Toledo RT. Fundamentals of Food Process Engineering. 2nd ed. New York, USA: Van Nostrand Reinhold; 1991.

16. Zareifard MR, Marcotte M, Dostie M. A method for balancing heat fluxes validated for a newly designed pilot oven. Journal of Food Engineering. 2006;76(3):303-312.
17. Salim EA, Abu-Goukh AA, Khalid HE, et al. Effect of drying method on spearmint (Mentha spicata var. viridis L.) oil content and physico chemical properties. American Journal of Phytomedicine and Cinical Therapeutics. 2015;3:1-7.

18. Baydar H, Erbas S. Effects of harvest time and drying on essential oil properities in Lavandin (Lavandula intermedia emeric ex losiel.). Acta horticulturae. 2009;826(826):377-382.

19. Venskutonis PR. Effect of drying on the volatile constituents of thyme (Thymus vulgaris L) and sage (Salvia officinalis L). Food Chemistry. 1997;59(2):219-227.

20. Charles DJ, Simon JE, Shock CC, et al. Effect of water stress and post-harvest handling on Artemisia content in the leaves of Artemisia апnи L. In: Janick J, et al. editors. New Crops, New York, USA: Wiley; 1993. p. 628-631.

21. Díaz-Maroto MC, Pérez-Coello MS, Cabezudo MD. Effect of drying method on the volatiles in bay leaf (Laurus nobilis L.). J Agric Food Chem. 2002;50(16):4520-4524.

22. Deans SG, Svoboda DP. Effect of drying regime on volatile oil and microflora of aromatic plants. Acta Horticulturae. 1992;306:450-452.

23. Díaz-Maroto MC, González Viñas MA, Cabezudo MD. Evaluation of the effect of drying on aroma of parsley by free choice profiling. Eur Food Res Technol. 2003;216(3):227-232.

24. Paul Boizot. Evaporation Rates of Essential Oils. UK: 14 Holly Bank Grove; 2014. 